\title{
Análisis de actitudes y autoeficacia percibida del profesorado ante la educación inclusiva
}

\author{
Alba Collado-Sanchis \\ Raúl Tárraga-Mínguez \\ Irene Lacruz-Pérez \\ Pilar Sanz-Cervera \\ Universitat de València. España. \\ cosanal@alumni.uv.es \\ raul.tarraga@uv.es \\ irene.lacruz@uv.es \\ pilar.sanz-cervera@uv.es
}

Recibido: 25/7/2019

Aceptado: 5/12/2019

Publicado: 20/7/2020

\section{Resumen}

La formación y la actitud del profesorado ante la inclusión educativa es un aspecto clave que debe ser estudiado teniendo en cuenta la elevada heterogeneidad de alumnado que actualmente se encuentra escolarizado en las aulas ordinarias. El objetivo de este estudio consiste en examinar las actitudes de los docentes hacia la inclusión de estudiantes con necesidades específicas de apoyo educativo en distintas etapas, además de analizar el nivel de autoeficacia percibida por parte de los mismos enseñantes. Teniendo en cuenta este objetivo, se administraron dos escalas, una para evaluar actitudes y otra para evaluar la autoeficacia percibida de 75 maestros. Además, también se realizaron entrevistas a algunos de los participantes, con el fin de obtener información cualitativa sobre su percepción de los procesos de inclusión. Los resultados mostraron diferencias entre los maestros de infantil y primaria frente a los profesores de secundaria, siendo la actitud de los docentes de los dos primeros niveles más positiva que la de los de enseñanza media. También se obtuvieron diferencias entre los docentes de primaria y secundaria con respecto a la autoeficacia percibida, siendo los maestros de primaria quienes se consideraban más preparados para atender las necesidades de este tipo de alumnado. Los resultados obtenidos muestran la necesidad de crear redes de apoyo colaborativo, además de desarrollar programas de formación docente.

Palabras clave: actitudes; autoeficacia; educación especial; educación inclusiva; formación del profesorado; necesidades específicas de apoyo educativo

\section{Resum. Anàlisi d'actituds i autoeficàcia percebuda del professorat davant l'educació inclusiva}

La formació i l'actitud del professorat davant la inclusió educativa és un aspecte clau que ha de ser estudiat tenint en compte l'elevada heterogeneïtat d'alumnat que actualment es troba escolaritzat a les aules ordinàries. L'objectiu d'aquest estudi consisteix a examinar les actituds dels docents cap a la inclusió d'estudiants amb necessitats específiques de suport educatiu en diferents etapes educatives, a més d'analitzar el nivell d'autoeficàcia percebuda 
per part dels mateixos ensenyants. Tenint en compte aquest objectiu, es van administrar dues escales, una per avaluar actituds i una altra per avaluar l'autoeficàcia percebuda de 75 mestres. A més, també es van realitzar entrevistes a alguns dels participants, amb la finalitat d'obtenir informació qualitativa sobre la seva percepció dels processos d'inclusió. Els resultats van mostrar diferències entre el professorat d'infantil i de primària enfront del de secundària, i l'actitud dels docents dels dos primers nivells va ser més positiva que la dels de l'ensenyament mitjà. També es van obtenir diferències entre els docents de primària i secundària en referència a l'autoeficàcia percebuda, sent els mestres de primària aquells que es consideren més preparats per atendre les necessitats d'aquest tipus d'alumnat. Els resultats obtinguts mostren la necessitat de crear xarxes de suport col-laboratiu, a més del desenvolupament de programes de formació docent.

Paraules clau: actituds; autoeficàcia; educació especial; educació inclusiva; formació del professorat; necessitats específiques de suport educatiu

\section{Abstract. Analysis of teachers' attitudes and perceived self-efficacy towards inclusive education}

Teachers' training and attitudes towards inclusive education are key aspects that merit attention given the large heterogeneity of students currently enrolled in mainstream classes. The aim of this study is to examine teachers' attitudes towards the inclusion of students with specific educational needs in different educational stages, in addition to the perceived level of self-efficacy of the teachers themselves. For this purpose, two scales were administered to 75 teachers, one to assess attitudes and the other to assess the teachers perceived self-efficacy. Moreover, some of the participants were interviewed in order to obtain qualitative information about their perception of processes of inclusion. The results showed some differences between early childhood and primary teachers compared to teachers working in secondary schools. Specifically, the attitude of teachers in the first two educational cycles was found to be more positive than that of secondary school teachers. Regarding perceived self-efficacy, differences were also observed between primary and secondary school teachers, as primary school teachers consider themselves to be more prepared to meet the needs of this type of students. The results demonstrate the need to create collaborative support networks and develop teacher training programmes.

Keywords: attitudes; inclusive education; self-efficacy; special education; specific educational support needs; teacher training

\section{Sumario}

$\begin{aligned} \text { 1. Introducción } & \text { 4. Discusión } \\ \text { 2. Método } & \text { 5. Conclusiones } \\ \text { 3. Resultados } & \text { Referencias bibliográficas }\end{aligned}$




\section{Introducción}

La diversidad es probablemente una de las características que actualmente mejor define nuestras aulas, un aspecto que supone una oportunidad para mejorar los procesos educativos, pero que también conlleva un desafío para todos los agentes implicados en el ámbito educativo.

Un pilar fundamental en el que podemos basarnos para superar dichos retos es el que aporta el movimiento de la educación inclusiva (Echeita y Duk, 2008), el cual incluye procesos que permiten incrementar la participación de todos los estudiantes conjuntamente, atendiendo no solo a aquellos etiquetados como alumnos con necesidades específicas de apoyo educativo (NEAE), sino también aspirando a realizar una atención individualizada, que tenga en cuenta las necesidades particulares de todos y cada uno de los estudiantes.

Esta concepción no contempla únicamente el hecho de impedir la exclusión dentro del sistema educativo, sino también fuera de él (UNESCO, 2005). Para conseguir este objetivo, es necesario tener una concepción más amplia, que no considere únicamente la necesidad de adaptar el currículum prefijado como única guía del proceso de enseñanza-aprendizaje.

Como señala Bandura (1987), esta concepción educativa no solo requiere la adquisición de nuevas destrezas docentes adaptadas a cada situación, sino que también reclama una profunda reflexión acerca de las prácticas de enseñanza, sobre la formación del profesorado y sobre las creencias pedagógicas de los profesionales.

En concreto, más allá de la necesaria formación del profesorado, las actitudes que los docentes posean respecto a la educación inclusiva es un aspecto clave que no solo condiciona el nivel de adaptación, sino que se ha comprobado que incluso incide directamente en el rendimiento escolar (BlotnickyGallant, Martin, McGonnell y Corkum, 2015).

La investigación sobre las actitudes de los docentes hacia la inclusión ha sido extensa en los últimos años (Chiner y Cardona, 2013; López, Almagro y Nicolás, 2017; Tárraga, Grau y Peirats, 2013; Tsakiridou y Polyzopoulou, 2014), mostrando que aunque las actitudes de los docentes generalmente son positivas, los mismos maestros afirman que en su práctica diaria encuentran muchas dificultades.

Se ha comprobado que la inclusión en las aulas es beneficiosa para todo el alumnado, pero es necesario contar con los recursos necesarios y con la formación adecuada (Akalin et al., 2014), considerando las propias fortalezas y determinación del profesorado para afrontar situaciones complejas y desafiantes (Barba-Martín, González-Calvo y Martínez-Scott, 2018).

Además, es necesario que exista una adecuada cultura institucional, ya que la inclusión no es un mero trabajo del tutor, sino que se trata de un deber de todo el sistema educativo (Darretxe, Goikoetxea y Fernández, 2013). Algunas investigaciones, en cambio, señalan que no todas las escuelas se preparan lo suficiente para conseguir la inclusión del alumnado en las aulas ordinarias (Shani y Hebel, 2016). 
La existencia de los maestros de educación especial como maestros de apoyo se considera una necesidad para conseguir el proceso de inclusión, teniendo en cuenta que estos están mejor formados que el profesorado generalista (Cardona, 2009), y que por ello pueden aportar una gran cantidad de recursos para atender al alumnado que presenta NEAE (Pegalajar y Colmenero, 2017).

Es muy importante que especialistas y tutores trabajen en colaboración, así como con otros posibles centros externos, con el fin de establecer una red de recursos (Hsieh, Hsieh, Ostrosky y McCollum, 2012; Savolainen, Engelbrecht, Nel y Malinen, 2012). No obstante, es posible que resulte complicado reunirse con maestros especialistas, debido a posibles limitaciones espaciotemporales.

Además de las actitudes, uno de los principales aspectos que también cabe considerar es el grado de autoeficacia que poseen los docentes, es decir, aquellos pensamientos que tiene el propio profesorado acerca de su capacidad para organizar y ejecutar los cursos de acción necesarios para conseguir determinados logros (Bandura, 1997).

En este caso concreto nos referimos a la capacidad que los propios docentes consideran que poseen para llevar a cabo una atención a la diversidad de calidad. Las investigaciones realizadas hasta el momento sobre el grado de autoeficacia que los docentes poseen concluyen que la mayoría se consideran ligeramente preparados para atender al alumnado con NEAE dentro del aula ordinaria (Marin, 2014).

Al realizar un análisis más exhaustivo según la etapa educativa, algunos estudios muestran que los docentes que pertenecen a las etapas de educación infantil y primaria se sienten más dispuestos a adaptar su práctica educativa a las necesidades de los niños con NEAE, en comparación con el profesorado de secundaria (Štemberger y Kiswarday, 2018). No obstante, el profesorado generalista, en ocasiones, muestra cierto rechazo ante el alumnado con NEAE que presenta comportamiento disruptivo (Nye et al., 2016).

Cabe considerar que la formación del profesorado junto a la experiencia profesional son aspectos clave que permiten aumentar la sensación de autoeficacia a la hora de dar respuesta a la diversidad presente en las aulas (Peebles y Mendaglio, 2014), de ahí la necesidad de incrementar la formación del profesorado.

Teniendo en cuenta que tanto las actitudes como el nivel de autoeficacia del profesorado son dos aspectos clave para hacer efectiva la inclusión, se plantea la necesidad de realizar un estudio en nuestro entorno cuyo principal objetivo sea valorar el concepto que tienen los docentes en activo en relación con la educación inclusiva que se lleva a cabo en los centros ordinarios.

Este objetivo general se concreta en dos objetivos específicos:

a) Comparar las actitudes hacia la inclusión educativa en una muestra de maestros y maestras de educación infantil, de educación primaria y de educación secundaria obligatoria. 
b) Comparar la percepción de autoeficacia para abordar la educación de estudiantes con NEAE en una muestra de maestros y maestras de las diferentes etapas educativas comentadas con anterioridad.

\section{Método}

Se empleó un diseño de investigación de tipo descriptivo transversal en que se aplicaron dos escalas a 75 participantes de tres centros educativos seleccionados por conveniencia (centros con un número significativo de estudiantes con NEAE). Se codificaron las respuestas de las escalas en el programa estadístico SPSS (versión 25) y se llevó a cabo un análisis de multivarianza. Adicionalmente, se realizaron entrevistas a cinco de los participantes también seleccionados por conveniencia (docentes con al menos cuatro estudiantes con algún tipo de NEAE en su aula). Las entrevistas fueron transcritas y se efectuó un análisis de contenido a partir de categorías previamente establecidas.

\subsection{Participantes}

En esta investigación participaron un total de 75 maestros de las diferentes etapas educativas ( 25 de educación infantil, 25 de educación primaria y 25 de educación secundaria obligatoria). Los participantes trabajaban en tres centros diferentes: dos centros públicos de educación infantil y primaria (uno de la ciudad de Valencia y otro de una localidad cercana a Valencia de alrededor de 8.000 habitantes) y un centro privado concertado que imparte desde la educación infantil hasta el bachillerato de una localidad del área metropolitana de Valencia de alrededor de 24.000 habitantes. Los tres centros escolarizan a estudiantes con NEAE y disponen de plan de atención a la diversidad de acuerdo con la normativa que establece la Conselleria de Educación de la Generalitat Valenciana.

Los 75 participantes cumplimentaron las escalas descritas en la sección de instrumentos. Adicionalmente se realizó una entrevista a cinco de los docentes (dos del centro privado concertado, dos del centro público de la localidad de mayor tamaño y uno del centro público de la localidad de menor tamaño). Los cinco entrevistados se seleccionaron debido a que en sus clases había escolarizados al menos cuatro estudiantes con alguna NEAE.

La media de edad de los encuestados fue de 39 años, con una experiencia profesional de entre 5 y 10 años. La mayoría había recibido formación específica en relación con la atención a la diversidad y había tenido experiencia con alumnado con NEAE.

La tabla 1 recoge las características demográficas básicas de los tres grupos de participantes en relación con el sexo y la edad, así como con los años de experiencia docente total, los años de experiencia docente con estudiantes con NEAE y si habían recibido formación en atención a la diversidad. 
Tabla 1. Información demográfica de los maestros participantes

\begin{tabular}{|c|c|c|c|c|}
\hline & & $\begin{array}{l}\text { Educación } \\
\text { infantil } \\
(N=25)\end{array}$ & $\begin{array}{c}\text { Educación } \\
\text { primaria } \\
(N=25)\end{array}$ & $\begin{array}{c}\text { Educación } \\
\text { secundaria } \\
\text { obligatoria } \\
(N=25)\end{array}$ \\
\hline \multirow{2}{*}{ Sexo } & Hombres & $6(24 \%)$ & $10(40 \%)$ & $9(36 \%)$ \\
\hline & Mujeres & 19 (76\%) & $15(60 \%)$ & $16(64 \%)$ \\
\hline \multicolumn{2}{|l|}{ Edad (DT) } & $39,4(9,02)$ & $33(10,43)$ & $44,56(10,46)$ \\
\hline \multirow{4}{*}{ Experiencia profesional } & $<5$ años & $5(20 \%)$ & $10(40 \%)$ & $4(16 \%)$ \\
\hline & 5-10 años & $7(28 \%)$ & $11(44 \%)$ & $5(20 \%)$ \\
\hline & 11-15 años & $9(36 \%)$ & $1(4 \%)$ & $7(28 \%)$ \\
\hline & $>15$ años & $4(16 \%)$ & $3(12 \%)$ & $9(36 \%)$ \\
\hline \multirow{4}{*}{$\begin{array}{l}\text { Experiencia profesional con } \\
\text { estudiantes con NEAE }\end{array}$} & $<5$ años & $9(36 \%)$ & $15(60 \%)$ & $6(25 \%)$ \\
\hline & 5-10 años & $10(40 \%)$ & $8(32 \%)$ & $7(29,2 \%)$ \\
\hline & 11-15 años & $4(16 \%)$ & $0(0 \%)$ & $6(25 \%)$ \\
\hline & $>15$ años & $2(8 \%)$ & $2(8 \%)$ & $5(20,8 \%)$ \\
\hline \multirow{2}{*}{$\begin{array}{l}\text { Formación en atención a la } \\
\text { diversidad }\end{array}$} & Sí & $22(88 \%)$ & $23(92 \%)$ & $5(20 \%)$ \\
\hline & No & $3(12 \%)$ & $2(8 \%)$ & $20(80 \%)$ \\
\hline
\end{tabular}

Fuente: elaboración propia.

\subsection{Instrumentos}

\subsubsection{Escala de actitudes hacia la inclusión (Tárraga, Grau y Peirats, 2013)}

Se trata de una escala de Likert de 23 ítems agrupados en 5 escalas. La primera de ellas evalúa los «beneficios de la inclusión" y corresponde a un total de 9 ítems (un ejemplo de este tipo de cuestiones sería: «La conducta de los estudiantes con NEAE es un mal ejemplo para sus compañeros/as sin NEAE»); el segundo grupo corresponde a un total de 4 ítems donde se valora la «atención generalista frente a la atención especialista» (e incluye cuestiones como: «El reto de estar en un aula generalista fomenta progreso académico de los estudiantes con NEAE»); el tercer factor se centra en la «metodología y manejo de la conducta en el aula» a través de 4 cuestiones (tales como: «El aumento de liberad en un aula generalista crea demasiada confusión para los estudiantes con NEAE»; el cuarto grupo habla sobre el «esfuerzo y dedicación del profesorado hacia los estudiantes con NEAE», y lo hace por medio de 3 enunciados (como, por ejemplo: «Los estudiantes con NEAE no monopolizan el tiempo que el profesor generalista dedica a sus estudiantes»), y, por último, la quinta agrupación hace referencia, a través de 3 ítems, a "la formación y competencia del profesorado» (formulados a través de cuestiones como: «Los maestros generalistas tienen la suficiente competencia profesional para trabajar con estudiantes con NEAE»).

La valoración de la escala se midió a través de 5 posibles respuestas, las cuales iban desde el Muy de acuerdo, que correspondía al número 5, pasando 
por De acuerdo (4), Ni de acuerdo ni en desacuerdo (3), En desacuerdo (2) y Totalmente en desacuerdo (1).

La puntuación del cuestionario oscila entre 23 y 92. La más elevada indica una actitud más favorable hacia la inclusión educativa.

\subsubsection{Teachers' Sense of Efficacy Scale (Tschannen-Moran y Hoy, 2001)}

Para realizar este estudio se hizo uso de la versión corta de dicho instrumento, la cual consta de un total de 12 ítems divididos en tres grupos: 1) compromiso del alumno, que incluye ítems como por ejemplo: «¿Cuánto cree que puede ayudar a que los estudiantes con NEAE valoren su aprendizaje?»; 2) prácticas instructivas, en las que encontramos ítems como, por ejemplo: «¿Cómo de bien puede implementar estrategias alternativas en el aula?», y 3) de gestión del aula, con preguntas tales como: "¿Hasta qué punto puede hacer para que el alumnado con NEAE siga las reglas del aula?».

La manera de evaluarlo fue a través de una escala de Likert con cinco posibles respuestas: 1 . Nada; 2. Muy poco; 3. Algo; 4. Bastante, y 5. Mucho.

La puntuación del cuestionario oscila entre 12 y 60 . La más elevada indica una mayor creencia de autoeficacia para alcanzar mayores niveles de inclusión educativa.

\subsubsection{Entrevistas sobre la percepción de la inclusión en el aula}

Además de las escalas sobre actitudes y autoeficacia, se realizaron un total de 15 entrevistas a diferentes profesionales ( 5 a maestros de educación infantil, 5 a maestros de educación primaria y 5 a profesores de educación secundaria). Las entrevistas fueron grabadas con previo aviso y bajo el consentimiento de los entrevistados para poder ser transcritas.

El objetivo de estas entrevistas fue realizar un análisis más cualitativo de algunas de las variables que pueden ser responsables (al menos en parte) de la generación de actitudes hacia inclusión y de la formación de un sentimiento de autoeficacia docente.

Concretamente, en cada entrevista se realizaron un total de siete preguntas en las que se pretendía conocer la valoración de los docentes sobre la manera en la que la Ley Orgánica para la mejora de la calidad educativa (Ley Orgánica 8/2013) aborda el tratamiento de las NEAE; la relación entre las funciones relacionadas con las NEAE que se otorgan al profesorado en el reglamento orgánico y funcional de los centros y los recursos de los que se disponen, y las metodologías y los materiales de enseñanza y su relación con la atención educativa de los estudiantes con NEAE.

Debido a las restricciones de espacio, en el presente trabajo únicamente se comentan los resultados de cuatro de estas preguntas, cuyos enunciados y categorías de análisis establecidas a priori se reflejan en la tabla 2. 
Tabla 2. Preguntas y categorías para el análisis de las entrevistas

\begin{tabular}{|c|c|}
\hline Preguntas & Categorías \\
\hline $\begin{array}{l}\text { 1. Tras la lectura de los artículos } 71 \text { y } 74 \text { de la LOMCE, que } \\
\text { exponen los principios de normalización, inclusión, no discri- } \\
\text { minación e igualdad efectiva en el acceso y la permanencia } \\
\text { en el sistema educativo, se solicita a los docentes una valo- } \\
\text { ración sobre el grado de cumplimiento de estos principios. }\end{array}$ & $\begin{array}{l}\text { a) Sí considera que los princi- } \\
\text { pios enunciados en la LOMCE } \\
\text { relacionados con las NEAE se } \\
\text { cumplen razonablemente. } \\
\text { b) Existen barreras que impiden } \\
\text { un cumplimiento razonable de } \\
\text { estos principios. }\end{array}$ \\
\hline $\begin{array}{l}\text { 2. Tras la lectura del artículo } 85 \text { del reglamento orgánico y } \\
\text { funcional de los centros educativos de la Comunidad Valen- } \\
\text { ciana, referido a las funciones del tutor, se solicita a los } \\
\text { docentes una valoración sobre los recursos de que disponen } \\
\text { para llevar a cabo todas esas funciones. }\end{array}$ & $\begin{array}{l}\text { a) Los tutores disponen de sufi- } \\
\text { cientes recursos para alcanzar a } \\
\text { realizar todas sus funciones. } \\
\text { b) La falta de recursos impide } \\
\text { que los tutores puedan desarro- } \\
\text { llar todas sus funciones. }\end{array}$ \\
\hline $\begin{array}{l}\text { 3. Tras el análisis de dos imágenes que representan una } \\
\text { metodología de enseñanza tradicional de transmisión de } \\
\text { conocimientos y una metodología en que el docente ejerce } \\
\text { de guía en los procesos de aprendizaje, se solicita a los } \\
\text { docentes su valoración de ambas metodologías y que auto- } \\
\text { evalúen en qué punto del continuo entre ambos extremos } \\
\text { metodológicos se sitúan. }\end{array}$ & $\begin{array}{l}\text { a) Se sitúa más cercano a la } \\
\text { metodología de transmisión de } \\
\text { conocimientos. } \\
\text { b) Se sitúa más cercano a la } \\
\text { metodología en que el docente } \\
\text { es un guía del proceso. }\end{array}$ \\
\hline $\begin{array}{l}\text { 4. Tras la lectura de una noticia de prensa cuyo titular es } \\
\text { "Unas madres celebran la expulsión de un niño con Asper- } \\
\text { ger del aula», se solicita a los docentes una reflexión sobre } \\
\text { el papel de las familias en la educación inclusiva. }\end{array}$ & $\begin{array}{l}\text { a) Las familias son un agente } \\
\text { que facilita los procesos de inclu- } \\
\text { sión de estudiantes con NEAE. } \\
\text { b) Existe resistencia por parte } \\
\text { de las familias para alcanzar los } \\
\text { objetivos de la inclusión de estu- } \\
\text { diantes con NEAE. }\end{array}$ \\
\hline
\end{tabular}

Fuente: elaboración propia.

\section{Resultados}

\subsection{Resultados derivados de las escalas de actitudes y de autoeficacia docente relacionada con la inclusión}

En relación con los resultados obtenidos en las escalas, el análisis multivariante realizado muestra diferencias estadísticamente significativas entre los grupos en el global de las puntuaciones (Lambda de Wilks $=0,658 ; F_{(14,132)}=2,20$; $\left.p=0,11 ; \eta^{2}=0,189\right)$.

Las tablas que se incluyen a continuación muestran el análisis de comparación por pares obtenido en las diferentes subescalas, tanto de la escala de actitudes (tabla 3) como de la escala de autoeficacia (tabla 4), mostrando las diferencias entre grupos en los casos en que estas son significativas.

En relación con el primero de los objetivos - comparar las actitudes del profesorado en las tres etapas educativas analizadas respecto a la inclusión-, se obtuvieron diferencias estadísticamente significativas en algunos de los grupos.

En concreto, se obtuvieron diferencias estadísticamente significativas en tres de las cinco subescalas que incluye el instrumento de actitudes. En dos de ellas, la atención de maestros generalistas frente a la atención de especialistas, así 
Tabla 3. Resultados de la escala de actitudes

\begin{tabular}{|c|c|c|c|c|c|c|c|c|}
\hline & & $\begin{array}{l}\text { Educación } \\
\text { infantil (EI) }\end{array}$ & $\begin{array}{l}\text { Educación } \\
\text { primaria } \\
\text { (EP) }\end{array}$ & $\begin{array}{c}\text { Educación } \\
\text { secundaria } \\
\text { obligatoria } \\
\text { (ESO) }\end{array}$ & $F_{(14,132)}$ & $p$ & $\eta_{p}^{2}$ & $\begin{array}{c}\text { Diferencias } \\
\text { entre } \\
\text { grupos }\end{array}$ \\
\hline \multirow{2}{*}{$\begin{array}{l}\text { Beneficios de la } \\
\text { inclusión }\end{array}$} & $M$ & 34,76 & 36,28 & 33,96 & \multirow[t]{2}{*}{$4,43^{*}$} & \multirow[t]{2}{*}{0,015} & \multirow[t]{2}{*}{0,110} & \multirow[t]{2}{*}{$\mathrm{EP}>\mathrm{ESO}$} \\
\hline & DT & 2,96 & 2,92 & 2,49 & & & & \\
\hline \multirow{2}{*}{$\begin{array}{l}\text { Atención genera- } \\
\text { lista versus aten- } \\
\text { ción especialista }\end{array}$} & $M$ & 13,28 & 15,84 & 13,12 & \multirow[t]{2}{*}{$9,78^{* *}$} & \multirow[t]{2}{*}{$<0,001$} & \multirow[t]{2}{*}{0,214} & \multirow{2}{*}{$\begin{array}{l}\mathrm{EP}>\mathrm{El}> \\
\mathrm{ESO}\end{array}$} \\
\hline & DT & 2,73 & 2,25 & 2,32 & & & & \\
\hline \multirow{2}{*}{$\begin{array}{l}\text { Metodología y } \\
\text { manejo de la } \\
\text { conducta }\end{array}$} & $\mathrm{M}$ & 13,60 & 14,20 & 13,60 & \multirow[t]{2}{*}{0,61} & \multirow[t]{2}{*}{0,546} & \multirow[t]{2}{*}{0,071} & \\
\hline & DT & 2,73 & 2,18 & 2,20 & & & & \\
\hline \multirow{2}{*}{$\begin{array}{l}\text { Esfuerzo y } \\
\text { dedicación del } \\
\text { profesorado hacia } \\
\text { estudiantes con } \\
\text { NEE }\end{array}$} & M & 8,60 & 9,92 & 8,84 & \multirow[t]{2}{*}{2,01} & \multirow[t]{2}{*}{0,142} & \multirow[t]{2}{*}{0,053} & \\
\hline & DT & 2,22 & 2,63 & 2,58 & & & & \\
\hline $\begin{array}{l}\text { Formación y } \\
\text { competencia del } \\
\text { profesorado }\end{array}$ & $\begin{array}{l}M \\
\text { DT }\end{array}$ & $\begin{array}{l}6,12 \\
1,74\end{array}$ & $\begin{array}{l}7,52 \\
2,54\end{array}$ & $\begin{array}{l}5,88 \\
1,81\end{array}$ & $4,62^{*}$ & 0,013 & 0,114 & $\begin{array}{l}\mathrm{EP}>\mathrm{El}> \\
\mathrm{ESO}\end{array}$ \\
\hline
\end{tabular}

Nota: ${ }^{*} p<0,05 ;{ }^{* *} p<0,01$

Fuente: elaboración propia.

Tabla 4. Resultados de la escala de autoeficacia

\begin{tabular}{lcccccccc}
\hline & & $\begin{array}{c}\text { Educación } \\
\text { infantil (EI) }\end{array}$ & $\begin{array}{c}\text { Educación } \\
\text { primaria } \\
\text { (EP) }\end{array}$ & $\begin{array}{c}\text { Educación } \\
\text { secundaria } \\
\text { obligatoria } \\
\text { (ESO) }\end{array}$ & $F_{(14,132)}$ & $p$ & $\eta_{p}^{2}$ & $\begin{array}{c}\text { Diferencias } \\
\text { entre } \\
\text { grupos }\end{array}$ \\
\hline $\begin{array}{l}\text { Compromiso del } \\
\text { profesorado }\end{array}$ & $M$ & 15,6 & 16,8 & 14,96 & $3,38^{*}$ & 0,040 & 0,086 & EP $>$ ESO \\
\hline Prácticas & $M$ & 1,94 & 2,16 & 3,31 & & & & \\
instructivas & $D T$ & 15,20 & 16,44 & 15,40 & 2,07 & 0,134 & 0,054 & \\
& 2,25 & 2,27 & 2,42 & & & & \\
Gestión del aula & $M$ & 14,68 & 16,08 & 14,64 & 2,01 & 0,141 & 0,053 & \\
\hline
\end{tabular}

Nota: * $p<0,05$

Fuente: elaboración propia.

como en la formación y competencia del profesorado, se obtuvieron diferencias estadísticamente significativas entre los tres grupos; mientras que en la subescala de beneficios de la inclusión encontramos diferencias significativas solamente entre el grupo de maestros de educación primaria y el grupo de profesores de educación secundaria obligatoria.

En cuanto al segundo de los objetivos, comparar la autoeficacia del profesorado en relación con la inclusión en las etapas educativas de infantil, primaria y secundaria, solamente se obtuvieron diferencias estadísticamente signifi- 
cativas entre los docentes de primaria y secundaria en el apartado denominado compromiso del profesorado.

\subsection{Resultados derivados de las entrevistas}

Tras analizar las entrevistas realizadas, extraemos los siguientes resultados:

El profesorado considera que existe una distancia evidente entre los principios que se enuncian en la LOMCE (Ley Orgánica 8/2013) y la realidad educativa de los centros. Hace hincapié en la ausencia de formación del profesorado y de recursos personales que permitan alcanzar una adecuada individualización de la enseñanza o que permitan implementar estrategias que verdaderamente faciliten los procesos de inclusión. A este respecto, un docente de segundo de educación primaria afirmaba lo siguiente:

Creo que esto debe ser así, debe ser como lo dice la ley, pero no es cierto que los centros educativos dispongan siempre de todos los recursos necesarios para alcanzar el máximo desarrollo personal, intelectual, social y emocional de los alumnos. Deben, por una parte, cuidar la formación del profesorado que está en las aulas y, por otra parte, también los recursos que el alumno necesita para estar en el aula ordinaria, que creo que es lo que debe de ser la inclusión $100 \%$ del alumno con NEAE. Estamos en el camino pero creo que aún quedan muchas cosas que hacer, tanto por parte de los profesores, que deben cambiar un poco su propio punto de vista, como por lo que nos ofrece la administración.

Respecto a las funciones que se encomiendan a los tutores, un docente de educación primaria comentaba lo siguiente:

Estamos muy escasos de materiales y tampoco estamos formados para atender las necesidades y orientar a las familias. Nos piden muchas cosas, pero a la hora de la verdad, no ofrecen medios.

En relación con las metodologías utilizadas, fueron llamativas las diferencias en las valoraciones entre el profesorado de infantil y primaria frente al de secundaria. Los maestros de los niveles inferiores afirmaban emplear metodologías innovadoras (aprendizaje cooperativo, rutinas de pensamiento visible, aprendizaje basado en proyectos), las cuales permiten al alumno acceder a los conocimientos de una manera más lúdica y siendo él mismo protagonista de su propio proceso de enseñanza-aprendizaje. Por el contrario, el profesorado de secundaria se mostró más reticente al uso de estas metodologías y comentó que, al ser alumnos de mayor edad, cuesta más mantener el orden e impiden la realización de modificaciones en la forma de impartir sus clases.

La comparación entre las afirmaciones que hacían al respecto un docente de educación primaria y uno de secundaria reflejan esta diferencia con bastante claridad: 
Docente de educación primaria: A mí no me gusta hablar mucho en clase. Yo, personalmente, soy de explicaciones cortas y además les anticipo el tiempo, 5 minutos, 10 minutos, 15 minutos según lo que necesite, lo novedoso que sea el contenido.

Docente de educación secundaria: La innovación de metodologías está bien, pero si tenemos que impartir todos los contenidos del curso, no hay tiempo para eso. Tienes que explicar el tema, hacer actividades y evaluar, y ya llegas justo a todo eso, así que es difícil probar cosas nuevas e ir cambiando.

Finalmente, todos los entrevistados creían en la necesidad de involucrar a las familias para conseguir una escuela más inclusiva. Muchos de los docentes comentaron la necesidad de formar a las madres y a los padres con el fin de sensibilizar y conseguir que comprendieran que el proceso de inclusión no debe darse solo en las aulas, sino que es la sociedad la que debe comenzar a cambiar. Es llamativo ver cómo un docente de educación primaria lo resumía del siguiente modo:

Yo creo que al igual que a los profesores nos tienen que formar y sensibilizar, a las familias del alumnado también, y creo que la sensibilización, la formación y la comprensión de estas situaciones es la base, porque luego lo que estos niños reciben en sus casas es lo que transmiten en el aula.

\section{Discusión}

El propósito de este estudio era examinar las actitudes de los maestros acerca de la educación inclusiva y conocer el grado de autoeficacia de los docentes en las diferentes etapas educativas obligatorias (infantil, primaria y secundaria).

Los resultados mostraron diferencias estadísticamente significativas en las percepciones de los docentes con respecto a la inclusión del alumnado con NEAE cuando se analizó el grado de enseñanza. Observamos cómo la actitud de los profesores de educación infantil y los de educación primaria son semejantes, mientras que surgieron mayores diferencias con respecto al profesorado de educación secundaria obligatoria.

En concreto, se obtuvieron diferencias estadísticamente significativas en las escalas I, II y V. A continuación, se incluye una breve discusión respecto a cada una de estas escalas.

En cuanto a la escala I, titulada «Beneficios de la inclusión», según las estadísticas, y como hemos comprobado en las investigaciones de Štemberger y Kiswarday (2018), los maestros de infantil y primaria consideran beneficioso que los estudiantes con NEAE estén dentro de las aulas ordinarias, ya que este hecho permite que se esfuercen más, además de que exista una mayor interacción entre todos los discentes del aula, fomentando la comprensión y la aceptación de los diferentes estudiantes. Los profesores de educación secundaria, en cambio, se muestran más reticentes ante este proceso, ya que consideran que su comportamiento puede llegar a ser un mal ejemplo para sus 
compañeros y afectar al resto de estudiantes. Esto mismo lo hemos visto reflejado en algunas de las investigaciones mencionadas anteriormente (Leyser, Zeiger y Romi, 2011; Nye et al., 2016).

En relación con la escala II, titulada «Atención generalista frente a atención especialista", la mayoría del profesorado considera que el aula ordinaria fomenta el progreso académico de aquellos alumnos con NEAE, lo que les permite desarrollar habilidades académicas y conseguir un efecto positivo en su desarrollo emocional. A pesar de esto, encontramos diferencias entre las tres etapas educativas analizadas, ya que las actitudes hacia la inclusión son más positivas en maestros de primaria, seguidos de los maestros de infantil y dejando al profesorado de secundaria en último lugar. Esto se ve apoyado por otras investigaciones llevadas a cabo, en las que se afirma que los docentes de enseñanzas inferiores muestran actitudes más positivas que los pertenecientes a grados superiores (Avissar, Reiter y Leyser, 2003).

En cuanto a la escala $V$, titulada «Formación y competencia del profesorado", la mayoría de los entrevistados mostraron interés en formarse para atender a la diversidad de las aulas. Aun así, no todo el profesorado consideraba que esta formación fuese suficiente, creyendo que los maestros especialistas podrían atender mejor a las necesidades del alumnado. En este caso el profesorado de primaria mostró una actitud más favorable, seguido de los maestros de infantil y el profesorado de secundaria, consecutivamente. Esta idea se podría ver reforzada en la investigación llevada a cabo por Avramidis y Kalyva (2007), la cual concluye que los docentes no muestran actitudes desfavorables hacia la inclusión, simplemente no encuentran las soluciones para responder a los problemas que consideran que están fuera de su control.

En cuanto al análisis de la autoeficacia percibida por parte de los docentes para atender al alumnado con NEAE, gran parte consideran que son capaces de responder correctamente ante las necesidades de este tipo de discentes.

No obstante, tan solo un pequeño porcentaje de profesores de educación secundaria, en comparación con los maestros de educación primaria, consideran que son capaces de hacer que los alumnos con NEAE crean que realizan bien su trabajo escolar. Probablemente, uno de los factores determinantes radica en la formación inicial, lo cual determina su actitud, su identidad y su práctica profesional (López-Torrijo y Mengual-Andrés, 2015).

$\mathrm{El}$ análisis de las respuestas de las entrevistas nos ayuda a identificar algunas de las barreras a la inclusión que detecta el profesorado. Entre estas barreras se encontraría la ausencia de formación y de recursos para hacer frente a los retos que plantea la inclusión, la sobrecarga de funciones de los tutores, uno de los agentes clave para facilitar estos procesos de inclusión y la necesidad de involucrar a las familias para que sean un agente que contribuya a facilitar la inclusión de todos los estudiantes a través de la transmisión a sus hijos de actitudes abiertas y positivas hacia la aceptación de las diferencias como un valor, no como una dificultad a superar. 
La realización de este trabajo incluye algunas limitaciones, como por ejemplo el tamaño muestral. Es posible que, quizá con un tamaño muestral mayor, se hubiesen podido obtener más diferencias entre los tres grupos que en este estudio no han alcanzado significación estadística. Otra limitación ha sido que tanto las escalas como las entrevistas empleadas para obtener información son instrumentos de autoinforme que no han sido complementados con procedimientos de observación en el aula, por lo que los resultados del estudio pueden haberse visto influenciados, en parte, por el sesgo de deseabilidad social.

Este estudio permite ser una apertura a posibles investigaciones futuras, en las que sería conveniente analizar si existen diferencias entre aquellos docentes que tienen formación previa frente a los que no, o comparar la actitud y la autoeficacia de aquellos que tienen más experiencia frente a los que tienen una experiencia menor.

\section{Conclusiones}

Los resultados del presente estudio nos llevan a alcanzar, al menos, tres conclusiones fundamentales:

En primer lugar, se ha constatado que existen diferencias entre el profesorado de educación infantil y primaria frente al profesorado de educación secundaria en cuanto a actitudes hacia la inclusión, sentimiento de autoeficacia para hacer frente a los retos que plantea la inclusión y uso de metodologías en el aula. Los resultados indican que, a mayor nivel educativo, mayores dificultades se encuentra el profesorado para poder hacer efectivo el principio de inclusión educativa.

En segundo lugar, concluimos que una de las barreras para la inclusión más relevante que han detectado los docentes participantes en el estudio es la necesidad de aumentar su propia formación para poder cumplir con los requisitos de un sistema escolar inclusivo.

Finalmente, concluimos que también existen barreras para la inclusión detectadas por el profesorado que guardan relación con la sobrecarga de tareas y la ausencia de recursos para hacer frente a los desafíos de la inclusión.

\section{Referencias bibliográficas}

Akalin, S.; Demir, S.; Sucuoglu, B.; Bakkaloglu, H. e Iscen, F. (2014). The Needs of Inclusive Preschool Teachers about Inclusive Practices. Eurasian Journal of Educational Research, 54, 39-60.

Avissar, G.; Reiter, S. y Leyser, Y. (2003). Principals' views and practices regarding inclusion: The case of Israeli elementary school principals. European Journal of Special Needs Education, 18(3), 355-369. $<$ http://dx.doi.org/10.1080/0885625032000120233>

Avramidis, E. y KalYva, E. (2007). The influence of teaching experience and professional development on Greek teachers' attitudes towards inclusion. European Journal of Special Needs Education, 22(4), 367-389.

<http://dx.doi.org/10.1080/08856250701649989> 
Bandura, A. (1987). Pensamiento y acción: Fundamentos sociales. Barcelona: Martínez Roca. Original de 1986: Social foundations of thought and action: A social cognitive theory. Englewood Cliffs (NJ): Prentice-Hall.

- (1997). Self-efficacy: The exercise of control. Nueva York: Freeman.

Barba-Martín, R.A.; González-Calvo, G. y Martínez-Scott, S. (2018). El papel de una maestra en la inclusión de una alumna sordociega en la comunidad educativa. Educar, 54(1), 83-99. <https://doi.org/10.5565/rev/educar.915>

Blotnicky-Gallant, P.; Martin, C.; McGonnell, M. y Corkum, P. (2015). Nova Scotia Teachers' ADHD Knowledge, Beliefs, and Classroom Management Practices. Canadian Journal of School Psychology, 30(1), 3-21.

Cardona, C.M. (2009). Teacher Education Students' Beliefs of Inclusion and Perceived Competence to Teach Students with Disabilities in Spain. Journal of the International Association of Special Education, 10(1), 33-41.

Chiner, E. y Cardona, M.C. (2013). Inclusive education in Spain: How do skills, resources, and supports affect regular education teachers' perceptions of inclusion? International Journal of Inclusive Education, 17(5), 526-541. <http://dx.doi.org/10.1080/13603116.2012.689864>

Darretxe, L.; Goikoetxea, J. y Fernández, A. (2013). Analysis of inclusive and exclusive practices in two schools in the basque country. Actualidades Investigativas en Educación, 13(2), 105-134. <http://dx.doi.org/10.15517/aie.v13i2.11734>

Echeita, G. y Duk, C. (2008). Inclusión educativa. REICE: Revista Iberoamericana sobre Calidad, Eficacia y Cambio en Educación, 6(2), 1-8.

Hsieh, W.Y.; Hsieh, C.M.; Ostrosky, M. y McCollum, J. (2012). Taiwanese firstgrade teachers' perceptions of inclusive education. International Journal of Inclusive Education, 16(1), 71-88.

<https://doi.org/10.1080/13603111003592283>

«Ley Orgánica 8/2013, de 9 de diciembre, para la mejora de la calidad educativa». Boletin Oficial del Estado, 295 (10 de diciembre). Recuperado de <www.boe. es/diario_boe/txt.php>.

Leyser, Y.; Z Ziger, T. y Romi, S. (2011). Changes in self-efficacy of prospective special and general education teachers: Implication for inclusive education. International Journal of Disability, Development and Education, 58(3), 241-255. <https://doi.org/10.1080/1034912X.2011.598397>

López, M.P.; Almagro, M.L.B. y Nicolás, B.G. (2017). Evaluación de las actuaciones generales del Plan de Atención a la Diversidad según la perspectiva del profesorado. Revista de Educación Inclusiva, 10(1), 147-160.

López-Torrijo, M. y Mengual-Andrés, S. (2015). An Attack on Inclusive Education in Secondary Education: Limitations in Initial Teacher Training in Spain. Journal of New Approaches in Educational Research, 4(1), 9. <https://doi.org/10.7821/naer.2015.1.100>

Marin, E. (2014). Are Today's General Education Teachers Prepared to Face Inclusion in the Classroom? Procedia-Social and Behavioral Sciences, 142, 702-707. <https://doi.org/10.1016/j.sbspro.2014.07.601>

Nye, E.; Gardner, F.; Hansford, L.; Edwards, V.; Hayes, R. y Ford, T. (2016). Classroom behaviour management strategies in response to problematic behaviours of primary school children with special educational needs: Views of special 
educational needs coordinators. Emotional and Behavioural Difficulties, 21(1), 43-60. <https://doi.org/10.1080/13632752.2015.1120048>

Peebles, J.L. y Mendaglio, S. (2014). The impact of direct experience on preservice teachers' self-efficacy for teaching in inclusive classrooms. International Journal of Inclusive Education, 18(12), 1321-1336. <https://doi.org/10.1080/13603116.2014.899635>

Pegalajar, M.C. y Colmenero, M.J. (2017). Actitudes y formación docente hacia la inclusión en Educación Secundaria Obligatoria. Revista Electrónica de Investigación Educativa, 19(1), 84-97. <https://doi.org/10.24320/redie.2017.19.1.765>

Savolainen, H.; Engelbrecht, P.; Nel, M. y Malinen, O.P. (2012). Understanding teachers' attitudes and self-efficacy in inclusive education: Implications for preservice and in-service teacher education. European Journal of Special Needs Education, 27(1), 51-68. <https://doi.org/10.1080/08856257.2011.613603>

Shani, M. y Hebel, O. (2016). Educating towards Inclusive Education: Assessing a Teacher-Training Program for Working with Pupils with Special Educational Needs and Disabilities (SEND) Enrolled in General Education Schools. International Journal of Special Education, 31(3), 3.

ŠTemberger, T. y KiswardaY, V.R. (2018). Attitude towards inclusive education: The perspective of Slovenian preschool and primary school teachers. European Journal of Special Needs Education, 33(1), 47-58. <https://doi.org/10.1080/08856257.2017.1297573>

Tárraga, R.; Grau, C. y Peirats, J. (2013). Actitudes de los estudiantes del Grado de Magisterio y del Máster de Educación Especial hacia la inclusión educativa. Revista Electrónica Interuniversitaria de Formación del Profesorado, 16(1), 55-72. $<$ https://doi.org/10.6018/reifop.16.1.179441>

Tsakiridou, H. y Polyzopoulou, K. (2014). Greek teachers' attitudes toward the inclusion of students with special educational needs. American Journal of Educational Research, 2(4), 208-218. $<$ https://doi.org/10.12691/education-2-4-6>

Tschannen-Moran, M. y Hoy, A.W. (2001). Teacher efficacy: Capturing an elusive construct. Teaching and Teacher Education, 17(7), 783-805. <https://doi.org/10.1016/S0742-051X(01)00036-1>

UNESCO (2005). Guidelines for Inclusion: Ensuring Acces to Education for All. Paris: UNESCO. 